\title{
Recapitulating orthotopic tumor model through establishment of a parotid gland tumor with lung metastasis using HeLa cell injection into nude mice
}

\author{
YOUNG-WOOK PARK ${ }^{1}$, SEONG-GON KIM ${ }^{1}$, JE-YONG CHOI $^{3}$ and SUK KEUN LEE ${ }^{2}$ \\ Departments of ${ }^{1}$ Oral and Maxillofacial Surgery, and ${ }^{2}$ Oral Pathology, College of Dentistry, Gangneung-Wonju \\ National University, Gangneung 210-702; ${ }^{3}$ Department of Biochemistry and Cell Biology, \\ School of Medicine, Kyungpook National University, Daegu 700-422, Korea
}

Received September 10, 2009; Accepted November 4, 2009

DOI: 10.3892/or_00000687

\begin{abstract}
The interaction between tumor cells and surrounding normal cells is very important in the prognosis of tumors. The object of this study was to determine the effect of recipient bed of tumor xenograft on tumor metastasis using a novel parotid gland tumor model. HeLa cells were xenografted into the parotid gland or subcutaneous tissues of athymic mice. Eight weeks after the transplantation, all mice were euthanized and specimens were immunostained with antibodies for angiogenic factors. FGF-2 was given to HeLa cells and the effect on the cellular migration was determined. EGF was also given to HeLa cells for the evaluation of FGF-2 induction. Immunohistochemical staining was done for the vascular metastasis-related factors on 26 human salivary gland tumors. HeLa cells showed significantly higher lung metastatic potential in the parotid gland than in the subcutis. Immunohistochemical staining revealed overexpression of FGF-2 in the parotid tumors compared to the subcutaneous tumors. The application of FGF-2 to implanting HeLa cells increased the cellular invasion in a dose-dependent manner. The application of EGF increases FGF-2 expression in HeLa cells. In clinical specimens, EGF and VEGF signaling proteins were more expressed than in normal salivary glandular tissues. In conclusion, a parotid tumor model of HeLa cells was successfully developed and it closely mimics high metastasis. These results indicate that recipient bed with intense EGF expression increases tumor metastasis through upregulation of FGF-2 expression.
\end{abstract}

\section{Introduction}

For understanding the process of cancer progression and metastasis, an appropriate animal model is essential. Most

Correspondence to: Dr Seong-Gon Kim, Department of Oral and Maxillofacial Surgery, College of Dentistry, Gangneung-Wonju National University, Gangneung 210-702, Korea

E-mail: epker@chol.com

Key words: salivary adenoid cystic carcinoma, vascular metastasis, orthotopic tumor model, VEGF signaling pathways current animal models consist of establishing subcutaneous tumors in athymic mice. However, subcutaneous tumors usually do not metastasize to regional lymph nodes or any distant site (1). Moreover, the organ microenvironment of the subcutis is certainly different from that of the organs where tumors originate. The metastatic lesions depend on multiple and complex interactions of metastatic cells with host tissues $(2,3)$. Different tumor microenvironments manifest in the different biological behavior of the tumors in orthotopic versus ectopic locations (4). Recently orthotopic tumor models have been successfully established which display metastatic patterns of the human tumors in the head and neck area $(5,6)$. Orthotopic tumor models allow the evaluation of treatment outcomes for metastatic lesions in a biological setting that recapitulate the disease process in humans. The set-up of the orthotopic tumor model, however, is difficult in some tumors. Since the salivary gland has abundant growth factors, the salivary gland can be a second choice for the xenograft site when the orthotopic model can not be set up.

For the metastasis of a tumor, angiogenesis is an essential step. Neoangiogenesis, the synthesis of new blood vessels, has been proposed as a critical step in tumor proliferation and vascular metastasis. Various angiogenic and anti-angiogenic cytokines and pathways have been characterized. Malignant salivary gland tumors overexpress the mitogenic and angiogenic proteins, fibroblast growth factor (FGF)-1, FGF-2 and FGF receptor (FGFR)-1 (7), and matrix metalloproteinase (MMP)-2 and MMP-9 were proposed as the invasion and metastasis-related factors in malignant tumors of the salivary glands (8). Normal salivary gland expresses the epidermal growth factor (EGF) and nerve growth factor (NGF) (9). The expression of the angiogenesis-related factors in the salivary gland tumor may be a result of the interaction between tumor cells and the interstitial tissue that expresses many growth factors. Because of the expression of EGF receptors (EGFR) in many kinds of tumors $(10,11)$, the abundant EGF may give a chance to tumor growth in the normal salivary gland. The expression of vascular endothelial growth factor (VEGF), interleukin-8 (IL-8) and FGF-2 are induced by the activation of EGFR (12). VEGF, IL-8 and FGF-2 are important in the tumor angiogenesis (12). 
Consistent with above reviews, we hypothesized that angiogenic signaling pathways are critical for the progression and metastasis of human cancer. If the interaction between tumor cells and the interstitial tissue is important in the production of the angiogenesis-related factor, xenografted tumors in the salivary gland may show higher expression of the angiogenesis-related factor than the xenografted tumors in the subcutis. Thereafter, the xenografted tumors in the salivary gland may show higher metastasis than the xenografted tumors in the subcutis. This study was designed to establish a salivary gland xenograft model, which should facilitate the molecular pathogenesis of metastasis.

\section{Materials and methods}

Reagents. Antibodies for immunohistochemical analysis were purchased as follows: polyclonal rabbit anti-EGF, anti-EGFR, anti-VEGF, anti-VEGFR-2 (Santa Cruz Biotechnology, Santa Cruz, CA, USA); anti-phosphorylated EGFR (pEGFR, Biosource International, Camarillo, CA, USA); antiphosphorylated VEGFR-2 and monoclonal mouse MMP-9 (pVEGFR-2/3; Oncogene, Cambridge, MA, USA); polyclonal rabbit anti-basic FGF (FGF-2, Sigma Chemical Co., St. Louis, MO, USA); rat anti-mouse CD31/PECAM (plateletendothelial cell adhesion molecule)-1 (CD31, BD PharMingen, San Diego, CA, USA). Secondary antibodies were peroxidaseconjugated goat anti-rabbit IgG and goat anti-mouse IgG (Jackson ImmunoResearch Laboratories, West Grove, CA, USA). Other reagents were $A B C$ reagent (Biocare Medical, Walnut Creek, CA, USA), 3,3'-diaminobenzidine (DAB; Research Genetics, Huntville, AL, USA), Gill's hematoxylin (Sigma Chemical Co.), FGF-2 and EGF (R\&D Systems, Minneapolis, MN, USA), and pepsin (Biomeda, Foster City, CA, USA).

Cell culture. HeLa cells were maintained as monolayer cultures in RPMI-1640 medium (Invitrogen, Carlsbad, CA, USA) supplemented with $15 \%$ fetal bovine serum, MDA1986 in DMEM (Invitrogen) supplemented with $10 \%$ fetal bovine serum containing L-glutamine, vitamins (Life Technologies, Inc., Grand Island, NY, USA), and penicillin-streptomycin (Flow Laboratories, Rockville, MD, USA). The cells were incubated in a mixture of $5 \% \mathrm{CO}_{2}$ and $95 \%$ air at $37^{\circ} \mathrm{C}$. The cultures were maintained for no longer than 12 weeks after recovery from frozen stocks.

Animals and orthotopic implantation of tumor cells. A 6-weekold male athymic nude mice were purchased from the Orient Bio Co., Seoul, Korea. The mice were used in accordance with the Animal Care and Use Guidelines of College of Dentistry, Kangnung-Wonju National University. The mice used for this study were 7 weeks old. To produce tumors, HeLa cells were harvested from subconfluent cultures. Only suspensions consisting of single cells with $>90 \%$ viability were used for the injections.

To induce anesthetic condition, the mice were intramusclularly injected with zolazepam $1.5 \mathrm{mg} / \mathrm{kg}$ (Zoletil ${ }^{\circledR}$; France) and xylazine hydrochloride $3.5 \mathrm{mg} / \mathrm{kg}$ (Rumpun ${ }^{\circledR}$; Bayer Korea, Korea). Differential numbers of $5 \times 10^{6}$ (Group 1),

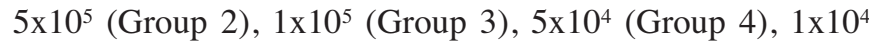
(Group 5), 1x10 (Group 6) cells were resuspended in $30 \mu 1$ of $\mathrm{Ca}^{2+}$ - and $\mathrm{Mg}^{2+}$-free Hanks' balanced salt solution (HBSS), and injected into the parotid gland using a 27-gauge hypodermic needle and a 1-ml syringe. A well-localized bleb confirmed a sussessful injection without leakage of the tumor cells. The preauricular incision was approximated in one layer using fine nylon sutures.

Subcutaneous implantation of tumor cells. In these groups, each mouse underwent subcutaneous injection of HeLa cells (suspended in a volume of $50 \mu 1$ ) directly into the right-side flank area with the same differential cell numbers.

Mice were then examined every other day for tumor development. The size of tumors was measured using calipers in cephalad-to-caudad and left-to-right dimensions. Tumor volumes was calculated using the formula: (large diameter) $\mathrm{x}$ (short diamter) ${ }^{2} / 2$ (13) and expressed in $\mathrm{mm}^{3}$. The body weights were recorded twice weekly.

Necropsy procedures and histologic examinations. Mice were euthanatized using anesthetics. Primary tumors in the parotid glands and in the subcutis were excised and weighed. For immunohistochemical and hematoxylin and eosin (H\&E) staining, one part of the tumor tissue was fixed in formalin and embedded in paraffin. Another part was embedded in OCT compound (Miles Inc., Elkhart, IN, USA), rapidly frozen in liquid nitrogen, and stored at $-70^{\circ} \mathrm{C}$. Cervical or inguinal lymph nodes and lungs were harvested, and the presence of metastatic lesion was confirmed by histological review.

Immunohistochemical determination of angiogenic factors on murine tumor tissues. To determine the level of expression of angiogenic factors, immunohistochemical staining for VEGF, FGF-2, MMP-9 and CD31 was performed using anti-VEGF, FGF-2, MMP-9 and CD31 antibodies. Paraffin-embedded tissues from murine parotid and subcutis tumors were prepared. For antigen retrieval, sections for MMP-9 was microwaved in water for $5 \mathrm{~min}$ followed by a 15-min cool down. Sections for VEGF and FGF-2 were incubated in pepsin for $20 \mathrm{~min}$ at $37^{\circ} \mathrm{C}$. Frozen tissues used for CD31 were sectioned (8$10 \mu \mathrm{m}$ ), mounted and air dried for $30 \mathrm{~min}$. Frozen sections were fixed in cold acetone for $5 \mathrm{~min}, 1: 1$ acetone/chloroform (v/v) for $5 \mathrm{~min}$ and acetone for $5 \mathrm{~min}$ and washed three times with PBS. Dilutions of primary antibodies were as follows: VEGF, 1:500; FGF-2, 1:1000; MMP-9, 1:50; and CD31, 1:800. Immunohistochemical procedures were done as described above. Negative controls were without primary antibodies.

Quantification of staining intensity of the angiogenic factors. For quantification of immunohistochemical reaction intensity, the absorbance of 100 positive cells of VEGF, FGF-2 and MMP-9 in 10 random $0.039-\mathrm{mm}^{2}$ fields at x200 magnification taken from either parotid or subcutis HeLa tumors, was evaluated by computer-assisted image analysis and expressed as mean density value. The samples were not counterstained so that the absorbance would be attributable solely to the product of the immunohistochemical reaction.

Quantification of microvessel density (MVD). For quantification of MVD, areas containing the higher number of 
Table I. Tumorigenicity, incidence of regional and distant metastasis.

\begin{tabular}{|c|c|c|c|c|c|c|}
\hline & \multicolumn{3}{|c|}{ Subcutis injection $(n=5)$} & \multicolumn{3}{|c|}{ Parotid injection $(n=5)$} \\
\hline & Primary tumor & Lymph nodes & Lung & Primary tumor & Lymph nodes & Lung \\
\hline Group $1\left(5 \times 10^{6}\right)$ & 5 & 0 & 0 & 5 & 0 & $1^{\mathrm{a}}$ \\
\hline Group $2\left(5 \times 10^{5}\right)$ & 5 & 0 & 0 & 5 & 0 & $2^{\mathrm{a}}$ \\
\hline Group $3\left(1 \times 10^{5}\right)$ & 3 & 0 & 0 & 3 & 0 & 0 \\
\hline Group $4\left(5 \times 10^{4}\right)$ & 2 & 0 & 0 & 3 & 0 & 0 \\
\hline Group $5\left(1 \times 10^{4}\right)$ & 0 & 0 & 0 & 0 & 0 & 0 \\
\hline Group $6\left(1 \times 10^{3}\right)$ & 0 & 0 & 0 & 0 & 0 & 0 \\
\hline
\end{tabular}

${ }^{\mathrm{a}} \mathrm{P}<0.05$.

tumor-related vessels were identified by scanning the tumor sections at $\mathrm{x} 40$ magnification. The vessels which were completely stained with anti-CD31 antibodies were then counted in 20 random $0.159-\mathrm{mm}^{2}$ fields at x100 magnification (14).

Cell migration assay and Western blotting for FGF-2. The cell migration was evaluated by the cell migration assay kit (Platypus Technologies, Madison, WI, USA). Briefly, HeLa cells were added to the well and incubated for cells to seed into outer annular region of the well. Then the insert was removed and the cells were allowed to migrate to the center of the well for $24 \mathrm{~h}$. The migration results were quantified by a plate reader. The control value that was not given FGF-2 was set as 100 . The value of the experimental group was shown as relative value to the control. For the Western blotting of FGF-2, EGF (10 ng/ml) was given to cultured HeLa cells and the supernatant was collected at $24 \mathrm{~h}$ after the application. The control was the cells grown without EGF. The subsequent procedure has been previously described (15).

Immunohistochemical analysis of EGF and VEGF signaling molecules in human salivary gland tumors. To determine the presence and the status of EGFR and VEGFR-2, 26 human salivary adenocystic carcinoma (ACC), which consisted of 18 ACCs with tubular-cribriform histologic pattern and 8 ACCs with solid pattern, were examined. All of the primary ACC samples were obtained from surgical specimens removed from patients who did not receive chemotherapy. Human salivary tumor specimens were evaluated for the presence of EGF, EGFR/pEGFR, VEGF and VEGFR-2/ pVEGFR-2. Formalin-fixed, paraffin-embedded specimens were sectioned at $4 \mu \mathrm{m}$ and mounted on positively charged slides and dried overnight. Adjacent normal salivary ducts, uninvolved by the tumor, served as internal control. Sections were deparaffinized in xylene, dehydrated with a graded series of alcohol, and rehydrated in PBS (phosphate-buffered solution). For antigen retrieval, sections for EGF, pEGFR, VEGFR-2 and pVEGFR-2 were microwaved in $10 \mathrm{mM}$ sodium citrate buffer ( $\mathrm{pH}$ 6.0; pEGFR in water) for $5 \mathrm{~min}$, and sections for EGFR and VEGF were incubated with pepsin for $20 \mathrm{~min}$ at $37^{\circ} \mathrm{C}$. Dilutions of primary antibodies were as follows: EGF, 1:100; EGFR, 1:200; pEGFR, 1:25; VEGF, 1:500; VEGFR-2, 1:200; and pVEGFR-2, 1:400. Indirect peroxidase technique for EGFR and VEGF and avidin-biotinylated peroxidase complex method for EGF, pEGFR, VEGFR-2 and pVEGFR-2 were used and a positive reaction was visualized by incubating the slides with DAB for 5-10 min. The sections were rinsed with distilled water, counterstained with Gill's hematoxylin and mounted with Universal Mount. Stained sections were examined in a Nikon Microphot-FX (Nikon Inc., Garden City, NY, USA) microscope. The intensity of protein labeling was evaluated on a semi-quantitative 4-point scale: -, no staining; +, weak staining; ++ , intermediate staining; +++ , strong staining.

Statistical analysis. Because of the relatively small sample size of our experiments, statistical methods based on standard maximum likelihood were not applicable. As an alternative, penalized logistic was used to assess the effects of parotid versus subcutis inoculation on tumorigenicity. The regression model included terms for the dilution effect and for the inoculation location (parotid versus subcutis). Group differences for continuous measurements were assessed via the non-parametric Kruskal-Wallis test or the non-parametric Wilcoxon rank sum test. Significance for the location effect was evaluated against an $\alpha$ significance level of 0.05 . Statistical analysis for comparing VEGF, FGF-2, MMP-9 staining and MVD between orthotopic and ectopic ACC tumors was done with the independent samples t-test.

\section{Results}

HeLa cells formed tumors when transplanted into the parotid glands of nude mice. When examined by necropsy 8 weeks after the injection of tumor cell suspension with differential cell numbers, Group 1-4 showed successful tumor formation (Table I, Fig. 1A). On histological examination, tissues of parotid glands were located at the periphery of the tumor mass, which means the tumor mass grew inside of the parotid glands (Fig. 1B).

Tumorigenicity and metastatic potential of HeLa cells in the parotid gland and in the skin. Compared with the skin tumors, the parotid tumors demonstrated no differences in the 


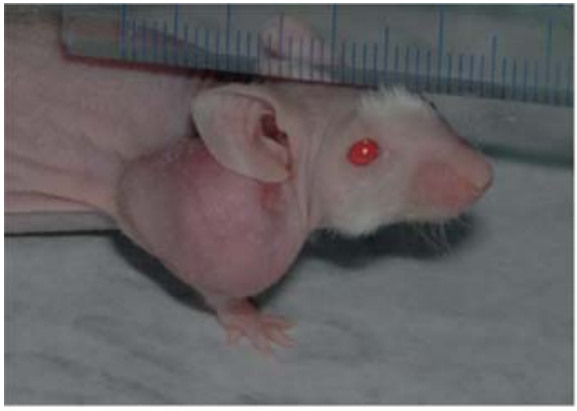

A

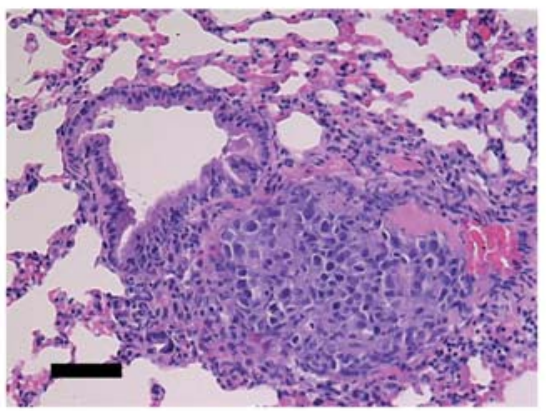

C

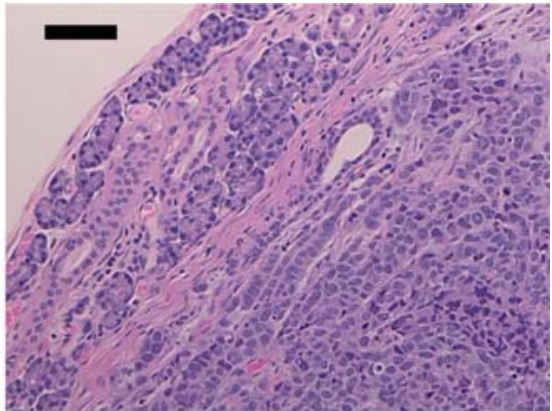

B

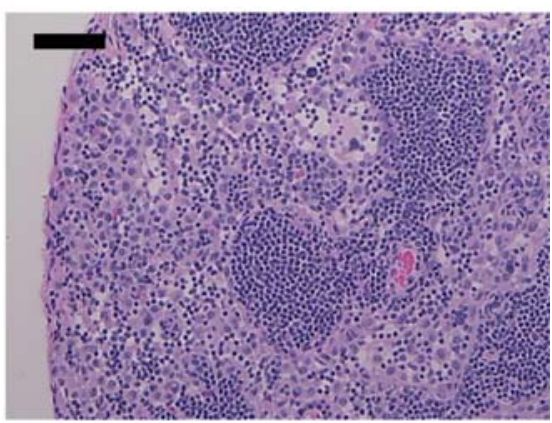

D

Figure 1. Features of the murine model of the salivary tumor. (A) Growth of the parotid tumor after implantation of HeLa cells into right parotid gland. (B) Implanted tumors were located inside the parotid gland tissues. Normal glandular tissues were displaced to the periphery of the tumor mass. (H\&E staining; Bars, $100 \mu \mathrm{m}$ ). (C) Near the small blood vessels in the lung, the metastatic tumor mass had developed, which means vascular metastasis. (H\&E staining; Bars, $100 \mu \mathrm{m}$ ). (D) Immune-related cells infiltrated into the nodal follicles. (H\&E staining; Bars, $100 \mu \mathrm{m}$ ).

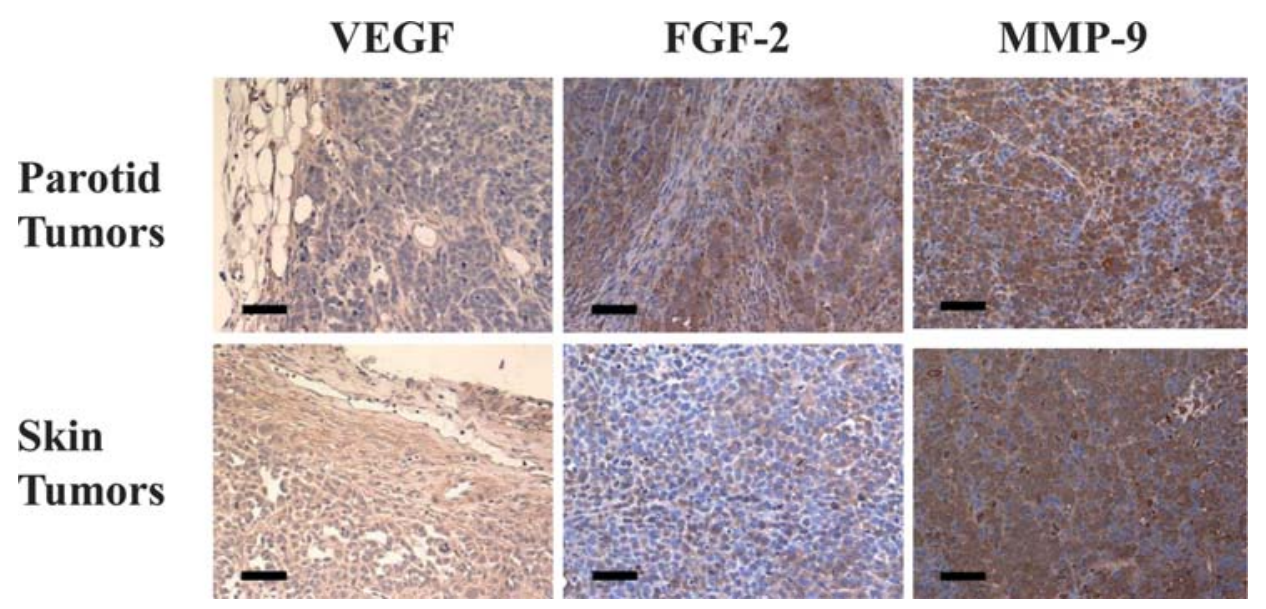

Figure 2. Expression of VEGF, FGF-2 and MMP-9 in the salivary gland and the skin tumors. Intensity of staining for FGF-2 was higher in the tumors implanted into the salivary gland than into the skin tumors. Representative results are shown. (Bars, $100 \mu \mathrm{m})$.

tumorigenicity $(\mathrm{P}>0.05$, Table I). However, the incidence of the lung metastasis was significantly higher when xenografted into the parotid glands (Table I, Fig. 1C). When dissected at necropsy, the cervical lymph node of the parotid tumor group had infiltrating cells proven to be immune-related cells, not metastatic tumor cells (Fig. 1D).

FGF-2 expression and MVD are higher in the parotid gland tumors than in the skin tumors. FGF-2 is known as a key player for tumor angiogenesis and metastasis $(7,14)$. Hence we determined whether FGF-2 expression was changed in our parotid gland tumor model. Immunohistochemical analysis showed that the parotid gland tumors expressed FGF-2 at higher levels than the skin tumors (Fig. 2). Moreover, MVD was significantly higher in the parotid gland tumors than in the skin tumors $(\mathrm{P}<0.05$, Table II $)$. The difference in the intensities of immunostaining for VEGF and MMP-9 were not statistically significant between the parotid gland tumors and the skin tumors ( $\mathrm{P}>0.05$, Fig. 2).

EGF-increased expression of FGF-2 and FGF-2 augments the cellular migration. When $10 \mathrm{ng} / \mathrm{ml}$ of EGF was applied 
Table II. Quantitative immunohistochemical analysis of the skin and the parotid tumors.

\begin{tabular}{llllr}
\hline & VEGF $^{\mathrm{a}}$ & FGF-2 $^{\mathrm{a}}$ & MMP-9 $^{\mathrm{a}}$ & MVD $^{\mathrm{b}}$ \\
\hline Parotid tumors & $0.21 \pm 0.08$ & $0.81 \pm 0.15^{\mathrm{c}}$ & $0.78 \pm 0.14$ & $13.8 \pm 3.2^{\mathrm{c}}$ \\
Skin tumors & $0.20 \pm 0.12$ & $0.37 \pm 0.22$ & $0.71 \pm 0.17$ & $7.6 \pm 2.8$ \\
\hline
\end{tabular}

${ }^{\mathrm{a}}$ Mean \pm SD absorbance determined as described in Materials and methods. ${ }^{\mathrm{b}}$ Microvessel density (MVD) was determined by measuring the number of completely stained blood vessels in 20 random $0.159-\mathrm{mm}^{2}$ fields at $\mathrm{x} 100$ magnification. ${ }^{\mathrm{C}} \mathrm{P}<0.05$ between the parotid and the skin tumor groups.

A

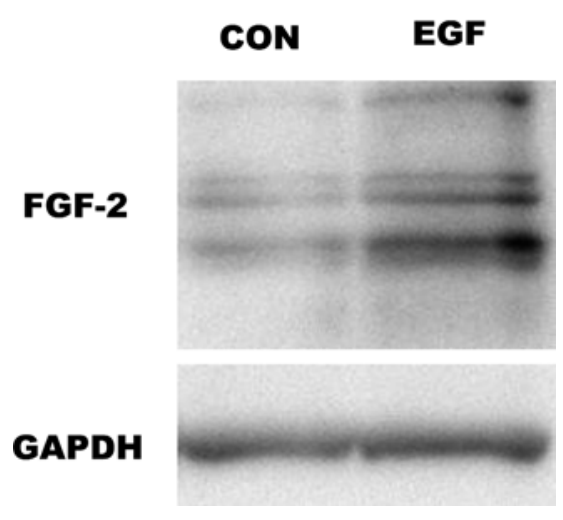

Figure 3. When $10 \mathrm{ng} / \mathrm{ml}$ of EGF was applied to HeLa cells, the expression of FGF-2 was increased (A). When exogenous FGF-2 was applied to the cells, the cellular migration was significantly increased $[\mathrm{P}<0.05,(\mathrm{~B})]$.

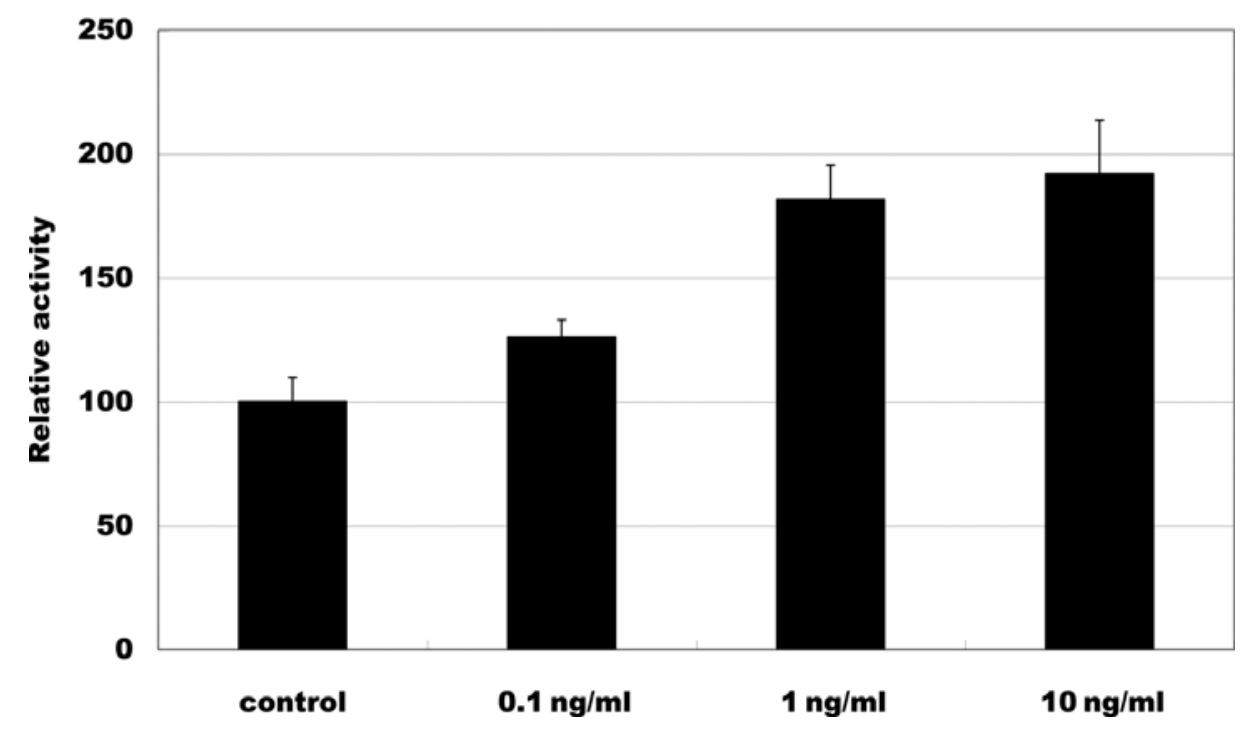

to HeLa cells, the expression of FGF-2 was increased (Fig. $3 \mathrm{~A})$. The cellular migration assay showed dose-dependent increase of the cellular migration. The relative activity of the control was $100.00 \pm 9.84$. When exogenous FGF-2 was applied to the cells, they were 126.14 $\pm 6.82(\mathrm{P}=0.019)$, $181.82 \pm 13.78(\mathrm{P}=0.001)$ and $192.05 \pm 21.65(\mathrm{P}=0.003)$ at 0.1 , 1 and $10 \mathrm{ng} / \mathrm{ml}$ of concentrations, respec-tively (Fig. 3B).
Human salivary ACC specimens express EGF signaling molecules. Many of the ACC tumors expressed all of these biomarkers. The overall percentages of the specimens expressing EGFR were $62 \%$ (16/26). Activated form of EGFR was also expressed in matching specimens. In addition, EGF immunoreactivity was found in both ACC tumor cells and normal salivary glands (Fig. 4). 


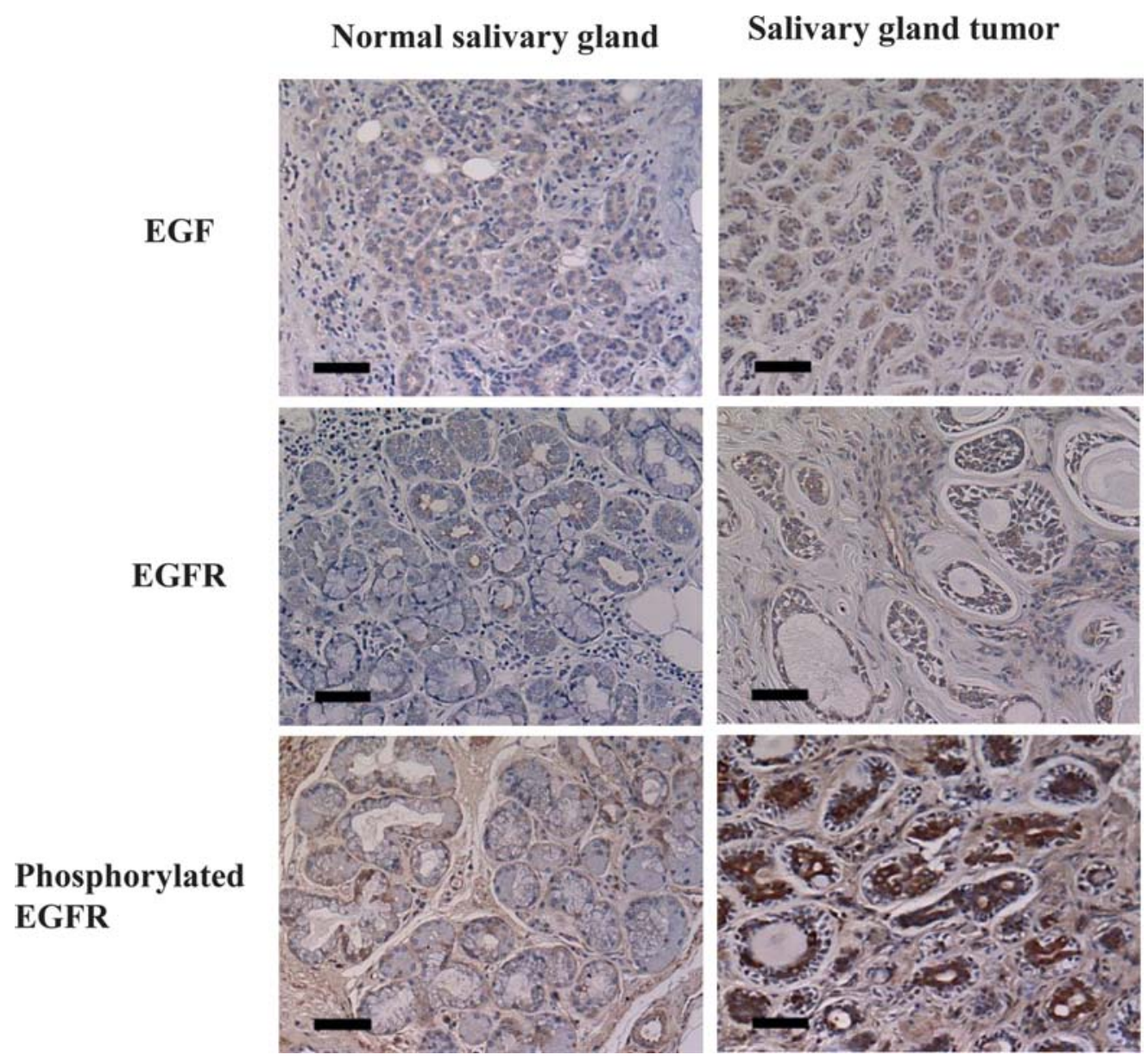

Figure 4. Immunohistochemical analyses for the expression of EGF and VEGF signaling molecules in human salivary adenoid cystic carcinoma tissues. Twenty-six surgical specimens were immunostained for expression of EGF, EGFR and phosphorylated (p) EGFR. Adjacent normal salivary ducts served as controls. Representative results are shown (Bars, $100 \mu \mathrm{m})$.

\section{Discussion}

We have established a parotid gland tumor model using HeLa cells showing a spontaneous lung metastasis without cervical nodal extension of tumor cells (Table I, Fig. 1). Furthermore, induced parotid gland tumors revealed higher expression of FGF-2 and microvessel density than subcutaneous tumors (Fig. 2). EGF expression was found in the normal salivary gland (Fig. 4). Both EGFR and pEGFR were expressed highly in the salivary ACC (Fig. 4). Exogenous EGF increased the expression of FGF-2 in HeLa cells (Fig. $3 \mathrm{~A})$. In addition, exogenous FGF-2 increased the migration of HeLa cells (Fig. 3B). Thus, the current work provided an animal model that is useful for the investigation of tumor metastasis and for the development of new treatment strategies.

Tumor metastasis is the ultimate display of complex interactions between tumor cells and the host microenvironments. It is a highly selective process and only a small portion of tumor cells form metastatic lesions in the secondary site (16). The implantation site influences the biological behavior of human tumor cells (16). Orthotopic implantation of tumor cells, which means implantation of tumor cells into the organ where tumors originate, can provide an in vivo model to investigate the biology and therapy of those tumors. In contrast with subcutaneous tumor models, orthotopic tumor models allow the evaluation of cancer metastasis and treatment strategies in a biological setting that mimics the disease process in humans. Orthotopic nude mouse models have been developed for a number of human malignancies including the lung (17), colon (18), tongue (5) and thyroid glands (6).

However, the setup of the orthotopic model is often difficult in some tumors. The size and the anatomical location are the limitation factors. Our parotid gland model exactly recapitulated the metastatic pattern of human tumors. Though HeLa cells originate from the uterus, they showed successful implantation into the parotid gland and showed higher metastasis than the tumor in the skin (Table I). The parotid gland model might have many advantages. The parotid gland expresses high level of EGF and NGF (9). Therefore, it will be highly useful to dissect tumorigenesis in case of EGF signal pathway-dependent cancer cells. The parotid gland has relatively large size and is easily accessed from minimal skin incision. The effects of the cytokines secreted by the parotid gland on the implanted tumor cells were clarified in this study.

The process of tumor metastasis is known to include the destruction of stroma and vascular basement membrane. The expression of FGF-2 in oral squamous carcinoma is higher 
than that in normal tissue (19). The expression level of FGF-2 in certain malignant tumor systems directly associated with invasion and metastasis (20). FGF-2 is also involved in the pro-angiogenic activity (21). The extent of angiogenesis is determined by the balance between positive and negative regulatory factors, which are produced by tumor cells as well as by host stromal cells. Activated macrophages influence the tumor-associated neoangiogenesis by secreting enzymes that can break down the extracellular matrix and by secreting angiogenic factors, such as FGF-2, platelete-derived growth factor (PDGF), insulin-like growth factor-1 and VEGF/VPF $(22,23)$. Therefore, FGF-2 activity can enhance tumor growth and survival, invasion, angiogenesis and metastasis.

The expression of FGF-2 in HeLa cells was higher in the parotid gland than in the skin (Fig. 2). The salivary gland secretes high level of EGF (9). The expression of EGF in the salivary gland was also confirmed in this study (Fig. 4). The exogenous EGF can bind to EGFR that localized on the cellular membrane (24). HeLa cells also express EGFR (25). EGFR has been proposed as a major target for cancer treatment because it is overexpressed in a variety of epithelial tumors $(10,11)$. The phosphorylated EGFR generated downstream signal and activates the transcription factor AP-1 $(26,27)$. Since FGF- 2 has an AP-1 binding site in its promoter, increased AP-1 activity results in FGF-2 upregulation (12). We showed that the exogenous EGF could induce the expression of FGF-2 in HeLa cells (Fig. 3A). The down-regulation of FGF-2 is subsequently shown to be due to the EGFR blocking in bladder carcinoma (12). The exogenous FGF-2 increased the cellular migration in a dosedependent manner (Fig. 3B). FGF-2 can stimulate the cellular migration in many cell types $(28,29)$. The release of FGF-2 into culture media is highest in metastasizing human cancer cell clones (30). However, the expression of FGF-2 in the normal salivary gland is limited mostly to the developmental period (31). Normal salivary gland does not express FGF-2 highly after birth (7). Therefore, the increased tumor metastasis of the parotid model might be due to FGF-2 overexpression in the tumor cells induced by exogenous EGF of the salivary gland.

Tumor xenografts in nude mice seldom infiltrate and metastasis (1). Since an orthotopically grafted tumor shows more metastases than a heterotopically transplanted tumor, the orthotopic graft model has been used for the metastasis model $(5,18)$. However, the orthotopic graft is highly technique-sensitive and some organs are very hard to approach surgically. The presented model might be used for any tumors that dependent on the EGF-related signal pathway for tumor cell proliferation and metastases. As the salivary gland is located beneath the skin, the surgical approach is very easy. Many tumors are known to express EGFR and their growth is dependent on the EGFR pathway. Tumor-associated vessels are also known to express EGFR $(32,33)$. Even, the primary cultured tumor cells grafted into the salivary gland were easily grown and metastasized (data not shown). Since tumor metastasis is important factor on the prognosis, the development of new cancer drug has been focused on control of tumor metastases. Therefore, our model will be very useful for drug testing. In conclusion, the salivary gland of nude mouse is an optimal place for the tumor xenograft and this model might be successfully applied not only to the study of the metastasis mechanism, but also to the cancer drug development and testing.

\section{Acknowledgements}

This study was supported by a grant of the Korea Health 21 R\&D Project, Ministry of Health and Welfare, Republic of Korea (A060174).

\section{References}

1. Hashitani S, Urabe M, Zushi Y, Segawa E, Okui S and Sakurai K: Establishment of nude mouse transplantable model of a human adenoid cystic carcinoma of the oral floor showing metastasis to the lymph node and lung. Oncol Rep 17: 67-72, 2007.

2. Fidler IJ: Seed and soil revisited. Contribution of the organ microenvironment to cancer metastasis. Surg Oncol Clin Nor Amer 10: 257-269, 2001.

3. Liotta LA and Kohn EC: The microenvironment of the tumourhost interface. Nature 411: 375-379, 2001.

4. Tseng W, Leong X and Engleman E: Orthotopic mouse model of colorectal cancer. J Vis Exp 10: 484, 2007.

5. Myers JN, Holsinger CH, Jasser SA, Bekele BN and Fidler IJ: An orthotopic nude mouse model of oral tongue squamous cell carcinoma. Clin Cancer Res 8: 293-298, 2002.

6. Kim S, Park YW, Schiff BA, et al: An orthotopic model of anaplastic thyroid carcinoma in athymic nude mice. Clin Cancer Res 11: 1713-1721, 2005.

7. Myoken Y, Myoken Y, Okamoto T, Sato JD, Kan M and McKeehan WL: Immunohistochemical study of overexpression of fibroblast growth factor-1 (FGF-1), FGF-2 and FGF receptor-1 in human malignant salivary gland tumours. J Pathol 178: 429-436, 1996.

8. Nagel H, Laskawi R, Wahlers A and Hemmerlein B: Expression of matrix metalloproteinases MMP-2, MMP-9 and their tissue inhibitors TIMP-1, -2 and -3 in benign and malignant tumours of the salivary gland. Histopathol 44: 222-231, 2004.

9. Knox SM and Hoffman MP: Salivary gland development and regeneration. In: Salivary Diagnostics. Wong DT (ed). WileyBlackwell, Ames, pp6-7, 2008.

10. Ettl T, Schwarz S, Kleinsasser N, Hartmann A, Reichert TE and Driemel O: Overexpression of EGFR and absence of C-KIT expression correlate with poor prognosis in salivary gland carcinomas. Histopathology 53: 567-577, 2008.

11. Kim JW, Kim HP, Im SA, et al: The growth inhibitory effect of lapatinib, a dual inhibitor of EGFR and HER2 tyrosine kinase, in gastric cancer cell lines. Cancer Lett 272: 296-306, 2008.

12. Perrotte $\mathrm{P}$, Matsumoto $\mathrm{T}$, Inoue $\mathrm{K}$, et al: Anti-epidermal growth factor receptor antibody $\mathrm{C} 225$ inhibits angiogenesis in human transitional cell carcinoma growing orthotopically in nude mice. Clin Cancer Res 5: 257-264, 1999.

13. Price JE: Xenograft models in immunodeficient animals. Metastasis Research Protocols. Vol 2. Brooks SA and Schumacher U (ed). Humana Press, Totowa, pp205-213, 2001.

14. Yoneda J, Kuniyasu H, Crispens MA, et al: Expression of angiogenesis-related genes and progression of human ovarian carcinomas in nude mice. J Natl Cancer Inst 90: 447-454, 1998.

15. Kim SG, Chae CH, Cho BO, et al: Apoptosis of oral epithelial cells in oral lichen planus caused by upregulation of BMP-4. J Oral Pathol Med 35: 37-45, 2006.

16. Fidler IJ: Rationale and methods for the use of nude mice to study the biology and therapy of human cancer metastasis. Cancer Metastasis Rev 5: 29-49, 1986.

17. Cui ZY, Park MJ, Lee J, et al: Modest anti-cancer activity of a bile acid acylated heparin derivative in a PC14PE6 induced orthotopic lung cancer model. Cancer Res Treat 41: 80-86, 2009.

18. Nakajima M, Morikawa K, Fabra A, Bucana CD and Fidler IJ: Influence of organ environment on extracellular matrix degradative activity and metastasis of human colon carcinoma cells. J Natl Cancer Inst 82: 1890-1898, 1990.

19. Myoken Y, Myoken Y, Okamoto T, Sato JD and Takada K: Immunocytochemical localization of fibroblast growth factor-1 (FGF-1) and bFGF in oral squamous cell carcinoma (SCC). J Oral Pathol Med 23: 451-456, 1994. 
20. Hase T, Kawashiri S, Tanaka A, et al: Correlation of basic fibroblast growth factor expression with the invasion and the prognosis of oral squamous cell carcinoma. J Oral Pathol Med 35: 136-139, 2006

21. Presta M, Dell'Era P, Mitola S, Moroni E, Ronca R and Rusnati M: Fibroblast growth factor/fibroblast growth factor receptor system in angiogenesis. Cytokine Growth Factor Rev 16: 159-178, 2005.

22. Koch AE, Polverini PJ, Kunkel SL, Harlow LA, DiPietro LA and Elner VM: Interleukin-8 as a macrophage derived mediator of angiogenesis. Science 258: 1798-1801, 1992.

23. Mantovani A, Bottazzi B, Colotta F, Sozzani S and Ruco L: The origin and function of tumor-associated macrophages. Immunol Today 13: 265-270, 1992.

24. Soufla G, Sifakis S and Spandidos DA: FGF2 transcript levels are positively correlated with EGF and IGF-1 in the malignant endometrium. Cancer Lett 259: 146-155, 2008.

25. Kharchenko MV, Aksyonov AA, Melikova MM and Kornilova ES: Epidermal growth factor (EGF) receptor endocytosis is accompanied by reorganization of microtubule system in HeLa cells. Cell Biol Int 31: 349-359, 2007.

26. Kizaka-Kondoh S, Sato K, Tamura K, Nojima H and Okayama H: Raf-1 protein kinase is an integral component of the oncogenic signal cascade shared by epidermal growth factor and plateletderived growth factor. Mol Cell Biol 12: 5078-5086, 1992.
27. Shen L, Shui Y, Wang X, et al: EGFR and HER2 expression in primary cervical cancers and corresponding lymph node metastases: implications for targeted radiotherapy. BMC Cancer 8: 232, 2008.

28. Bikfalvi A, Klein S, Pintucci G and Rifkin DB: Biological roles of fibroblast growth factor-2. Endocr Rev 18: 26-45, 1997.

29. Powers CJ, McLeskey SW and Wellstein A: Fibroblast growth factors, their receptors and signaling. Endocr Relat Cancer 7: 165-197, 2000

30. Azuma M, Yuki T, Motegi K and Sato M: Enhancement of bFGF export associated with malignant progression of human salivary gland cell clones. Int J Cancer 71: 891-896, 1997.

31. Patel VN, Rebustini IT and Hoffman MP: Salivary gland branching morphogenesis. Differentiation 74: 349-364, 2006.

32. Amin DN, Hida K, Bielenberg DR and Klagsbrun M: Tumor endothelial cells express epidermal growth factor receptor (EGFR) but not ErbB3 and are responsive to EGF and to EGFR kinase inhibitors. Cancer Res 66: 2173-2180, 2006.

33. Kim SJ, Uehara H, Karashima T, Shepherd DL, Killion JJ and Fidler IJ: Blockade of epidermal growth factor receptor signaling in tumor cells and tumor-associated endothelial cells for therapy of androgen-independent human prostate cancer growing in the bone of nude mice. Clin Cancer Res 9: 1200-1210, 2003. 